\title{
СРАВНИТЕЛЬНЫЙ АНАЛИЗ МЕТОДОВ ИМИТАЦИОННОГО И АНАЛИТИЧЕСКОГО МОДЕЛИРОВАНИЯ В ТЕПЛОФИЗИКЕ ГОРНЫХ ВЫРАБОТОК
}

\author{
Б.П. Казаков \\ Горный институт УрО РАН, г. Пермь
}

\begin{abstract}
Аннотация: Проведён анализ методов имитационного и аналитического моделирования теплообменных процессов в атмосфере горных выработок. Рассмотрены три модели: на основе введения коэффициента нестационарного теплообмена, решение задачи в полусопряжённой постановке с помощью преобразований Лапласа и численное решение методом конечных объёмов. Показано, что зависимости первой модели являются наиболее широко используемыми как в оценочных инженерных расчётах, так и в составе специализированных программ, т.к. не требуют больших затрат времени и вычислительных ресурсов. Однако установлено, что при больших временах теплообмена порядка 10 лет расчётное значение температуры воздуха с использованием коэффициента нестационарного теплообмена оказывается завышенным в несколько раз. Вторая модель позволяет получить более точное аналитическое решение дифференциальных уравнений теплообмена в квадратурах. Имитационное моделирование является наиболее детальным и точным и отличается вариативностью постановочных условий. Выявлены недостатки каждого из методов, к числу которых отнесены плохая адаптация к добавлению осложняющих факторов для аналитических и большая вычислительная ресурсоёмкость для имитационных методов. На основании проведённого сравнительного анализа методов моделирования теплообменных процессов в шахтной теплофизике сделано заключение, что аналитические и имитационные методы дополняют друга, поэтому оптимальным является их комплексное использование с целью извлечения преимуществ обоих методов.
\end{abstract}

Ключевые слова: теплопроводность, ресурсоёмкость, воздухоподающий ствол, коэффициент нестационарного теплообмена, влагообмен, преобразования Лапласа, метод конечных объёмов.

\section{Введение}

С развитием компьютерных технологий появляется всё больше возможностей для замены ручной работы по моделированию физических процессов вычислительными комплексами, позволяющими решать широкий класс задач без проведения аналитических исследований. Используются известные программные продукты типа ANSYS, Comsol или SolidWorks, которые по заданным исходным начальным и граничным условиям выдают готовые результаты. Либо составляются дифференциальные уравнения, отражающие поведение моделируемой системы в максимально общей постановке, которые решаются с помощью стандартных численных методов. В недалёком прошлом такой подход был неприемлем, поскольку мощность компьютеров не позволяла решать задачи с такой степенью детализации, необходимо было искать упрощения, тщательно анализируя все механизмы и выявляя наиболее значимые из них. То же можно сказать и о выборе математических методов решения - в приоритете были аналитические методы, дающие возможность получить конечный численный результат, а не ограничиваться написанием системы уравнений, «неподъёмной» для численного решения.

Несомненно, повышение производительности научного труда за счёт использования компьютерных вычислений является неотъемлемой частью научно-технического прогресса. Имитационное моделирование значительно расширяет горизонт моделируемых явлений, увеличивает степень их детализации и взаимосвязанности частей. Его практическая значимость постоянно возрастает, и, как результат, падает популярность аналитических методов моделирования, которые являются более трудоёмкими и менее эффективными. Ввиду постоянно возрастающей мощности компьютеров вычислительная ресурсоёмкость методов уже не так актуальна, как раньше, и отступает на второй план. Бытует мнение, что аналитические модели являются пережитком прошлого, поэтому 
всё чаще в научных работах фигурируют результаты, полученные исключительно методом «подставили - получили», со ссылками на авторитетные вычислительные пакеты. Несмотря на очевидные преимущества высокоточной имитации перед упрощёнными моделями, некоторые важные особенности аналитических методов в имитации отсутствуют. Прежде всего, это возможность раздельного описания отдельных механизмов модели, что значительно увеличивает их наглядность и аналитическую прозрачность в теоретическом плане. Рост производительности компьютерной техники сопровождается не менее значительным ростом потребности в решении всё более сложных ресурсоёмких задач оптимизации, автоматизации, управления, которые оказываются слишком затратными для решения без упрощений. Кроме того, на практике востребованы не столько очень точные, сколько простые в использовании методы решения производственных задач, для которых не требуются суперкомпьютеры, специализированное программное обеспечение и специалисты, умеющие им пользоваться. Поэтому и прикладная значимость аналитических методов моделирования по-прежнему сохраняется.

Преимущества и недостатки методов аналитического и имитационного моделирования демонстрируются далее на примерах математического описания теплообменных процессов между рудничным воздухом и породным массивом. Задачи рудничного теплообмена актуальны, прежде всего, тем, что их решение позволяет прогнозировать изменения теплофизических параметров воздуха при его движении по горным выработкам. С другой стороны, информация об изменениях температуры породного массива при тепловом контакте с шахтным воздухом также востребована, например, о скорости охлаждения крепи вентиляционного ствола при аварийном реверсировании вентилятора в холодный период года.

\section{Аналитический метод на основе введения модельной характеристики - коэффициента нестационарного теплообмена}

Необходимость математического моделирования и расчёта теплообмена рудничного воздуха с горным массивом возникла достаточно давно, когда возможности вычислительной техники были ещё недостаточны для численного решения задач подобного рода. Поэтому исторически сложилось так, что от сопряжённой постановки отказались, и на первый план вышла упрощённая расчётная модель. Суть упрощения заключается в определении потока тепла $q=q(t, z), \mathrm{BT} / \mathrm{m}^{2}$ на границе воздуха с породным массивом в виде известной функции времени $t$, с и координаты $z$, м, после чего задача теплообмена распадается на две независимые подзадачи, отдельно для воздуха и для массива. Поток $q(t, z)$ пропорционален разнице температур $T_{1}(t, z),{ }^{\circ} \mathrm{C}$ и $T_{2}(t, z),{ }^{\circ} \mathrm{C}$ на границе раздела массива и воздуха, но в качестве упрощения делается следующая замена: $q(t, z)=\alpha\left(T_{1}(t, z)-T_{2}(t, z)\right) \rightarrow q(t, z)=k_{t}\left(T_{1}^{\infty}-T_{2}(t, z)\right)$, где $\alpha, \mathrm{BT} /\left(\mathrm{M}^{2} \cdot{ }^{\circ} \mathrm{C}\right)-$ коэффициент теплоотдачи, $T_{1}^{\infty}$, ${ }^{\circ} C$ - температура массива на бесконечности, а функция $T_{1}(t, z)$ включена в состав коэффициента пропорциональности, который назван коэффициентом нестационарного теплообмена [1].

Способы вычисления $k_{t}, \mathrm{BT} /\left(\mathrm{M}^{2} \cdot{ }^{\circ} \mathrm{C}\right)$ различны. Если задача плоская и нет зависимости от $z$, то $k_{t}$ вычисляется точно: $k_{t}=\frac{\kappa}{\sqrt{\pi \chi t}+\kappa / \alpha}$, где $\chi$ и $\kappa-$ температуропроводность, $\mathrm{m}^{2} / \mathrm{c}$ и теплопроводность, Вт/(м $\left.{ }^{\circ} \mathrm{C}\right)$ массива. В более сложных случаях $k_{t}$ определяется приближённо с помощью модельных упрощений.

1) Щербань А.Н. Зависимость получена для выработок, проветриваемых свыше 1 года, на основе решения плоской задачи [2]: 


$$
k_{t}=\frac{1}{1+\frac{\kappa}{2 \alpha R}}\left[\frac{\kappa}{2 R}+\frac{\kappa}{\sqrt{\pi \chi t}\left(1+\frac{\kappa}{2 \alpha R}\right)}\right],
$$

где $R$ - радиус выработки, м.

2) Воропаев А.Ф. Формула получена также на основе решения плоской задачи, проведено усреднение по времени изменения температуры воздуха:

$$
k_{t}=\frac{\kappa\left(1+0.27 \sqrt[4]{\frac{\chi t}{R^{2}}}\right)}{0.88 \sqrt{\chi t}+\kappa / \alpha} F\left(F_{O}, B i\right)
$$

где $F o \equiv \frac{\chi t}{R^{2}}$ и $B i \equiv \frac{\alpha R}{\kappa}-$ критерии Фурье и Био. Величина $F$, по утверждению автора, близка к единице в интервалах времени $t$ от 1 часа до 50 лет [3].

3) Лужецкая Н.Д. Зависимость получена решением двумерной краевой задачи теплопроводности Фурье для полого полубесконечного цилиндра с разной радиальной теплопроводностью (по $x$, м по $y$, м):

$$
k_{t}=\kappa_{x}\left\{\frac{1}{2 R \sqrt{1-\left(1-\chi_{x} / \chi_{y}\right)^{2}}}+\frac{1}{\sqrt{\pi \chi_{x} t}} \frac{2}{\pi} \int_{0}^{\pi / 2} \frac{d \varphi}{\sqrt{1-\left(1-\chi_{x} / \chi_{y}\right) \sin ^{2} \varphi}}\right\} .
$$

Приведенные формулы (1)-(2)-(3) ввиду своей простоты являются наиболее широко используемыми для расчёта величины коэффициента нестационарного теплообмена, как в оценочных инженерных расчётах, так и в составе специализированных программ. Получение расчётных количественных результатов не требует больших затрат времени и вычислительных ресурсов. К недостаткам следует отнести «негибкость» моделей по отношению к различным осложняющим факторам и невозможность точной оценки модельной погрешности на малых и больших интервалах времени.
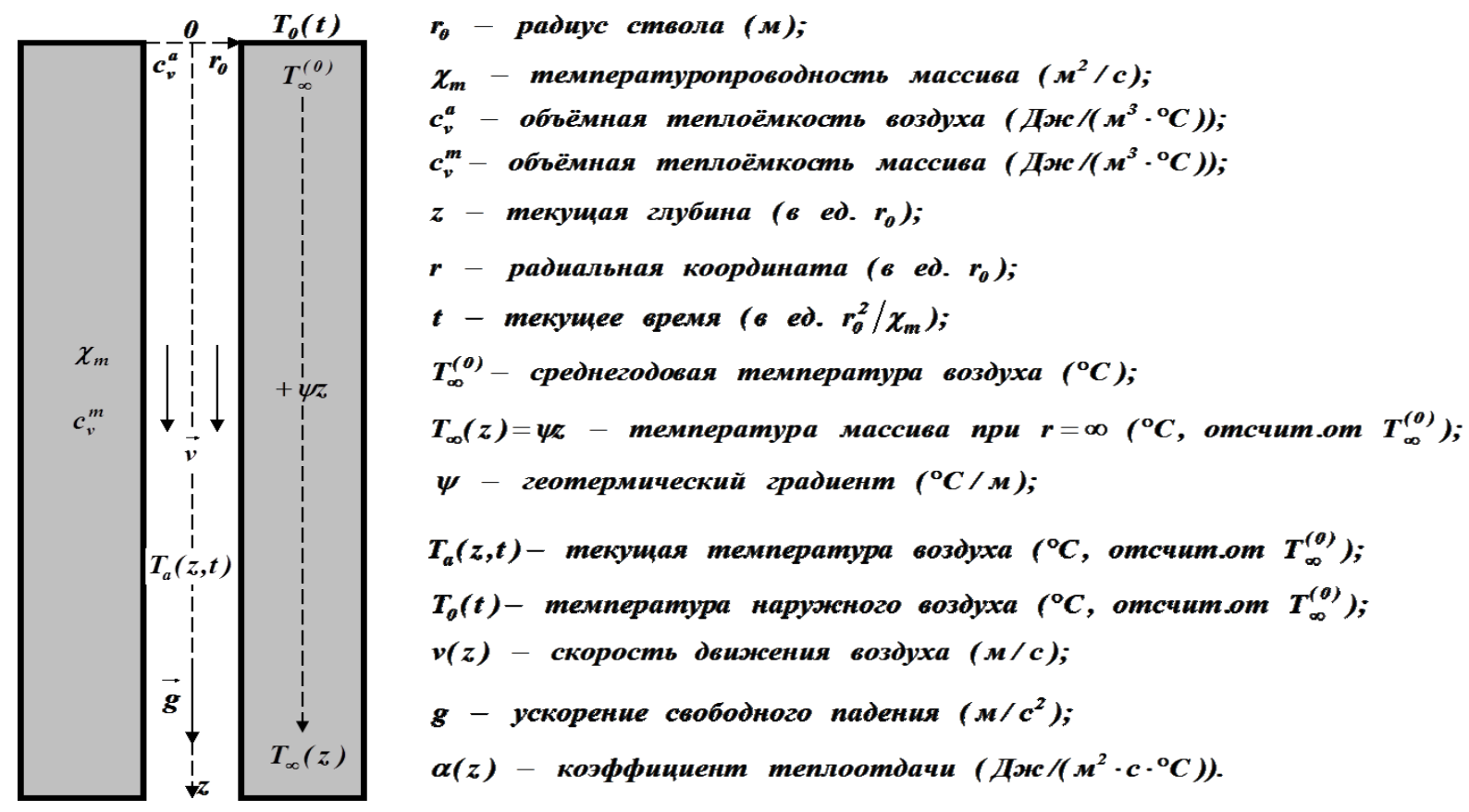

Рис. 1. Теплообмен вентиляционного воздуха с породным массивом в стволе 


\section{Аналитическое моделирование теплообмена с помощью преобразований Лапласа}

С целью получения более точных и адекватных реальности результатов, возможности моделирования быстрых и длительных теплообменных процессов, задача решена в полусопряжённой постановке без использования $k_{t}$ на примере теплообмена в воздухоподающем стволе (рис. 1) [4, 5].

$$
\left\{\begin{array}{l}
\text { 1. } \frac{\partial T_{m}}{\partial t}=\frac{1}{r} \frac{\partial}{\partial r}\left(r \frac{\partial T_{m}}{\partial r}\right)-\text { уравнение теплопроводности в массиве; } \\
\text { 2. }\left(a(z) \frac{\partial T_{a}}{\partial z}+\frac{\partial T_{a}}{\partial t}\right)_{r=1}=\left.b \frac{\partial T_{m}}{\partial r}\right|_{r=1}-\text { баланс теплосодержсания в воздухе; } \\
\text { 3. }\left.\left(\frac{c(z)}{b} T_{m}-T_{a}\right)\right|_{r=1}=\left.\frac{\partial T_{m}}{\partial r}\right|_{r=1}-\text { скачок температуры на границе; } \\
\text { 4. } T_{a}(r=1, t, z=0)=T_{0}(t)-\text { температура воздуха на поверхности; } \\
\text { 5. } T_{a}(r, t=0, z)=T_{m}(r, t=0, z)=0 \text { пачальное условие. }
\end{array}\right.
$$

Безразмерные комплексы: $\quad a(z)=\frac{v(z) r_{0}}{\chi_{m}}, \quad b=\frac{2 c_{v}^{m}}{c_{v}^{a}}, \quad c(z)=\frac{2 \alpha(z) r_{0}}{c_{v}^{a} \chi_{m}}$.

Коэффициент теплоотдачи при $\operatorname{Re}>10^{4}: \alpha(z)\left(\frac{\text { Джс }}{M^{2} \cdot c \cdot{ }^{\circ} \mathrm{C}}\right)=1.7 \frac{v(z)(M / c)^{0.8}}{r_{o}(M)^{0.2}}$.

Система уравнений теплопроводности в цилиндрических координатах для неограниченного массива и баланса теплосодержания в движущемся воздухе с граничным условием третьего рода (4) решается операционным методом [6], позволяющим получить аналитическое решение для функции-изображения температуры воздуха $\tau_{a}$ и массива $\tau_{m}$, выраженные через функции Бесселя (5). Несмотря на громоздкость, решение всё же является более простым и менее ресурсоёмким для численной реализации, чем сеточные методы имитационного моделирования. Оно позволяет также оценить погрешность моделей с коэффициентом нестационарного теплообмена.

$$
\begin{aligned}
& \tau_{a}(p, z)=\exp \left\{-\int_{\theta}^{z} \frac{\omega(\zeta) d \zeta}{a(\zeta)}\right\}\left(\tau_{\theta}(p)+\frac{\psi}{p} \int_{\theta}^{\bar{z}} \frac{\omega(\zeta)}{a(\zeta)} \zeta \exp \left\{\int_{\theta}^{\zeta} \frac{\omega(\xi) d \xi}{a(\xi)}\right\} d \zeta\right)
\end{aligned}
$$

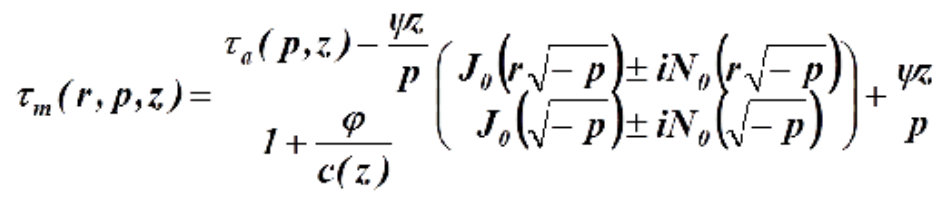

Обюзначения :

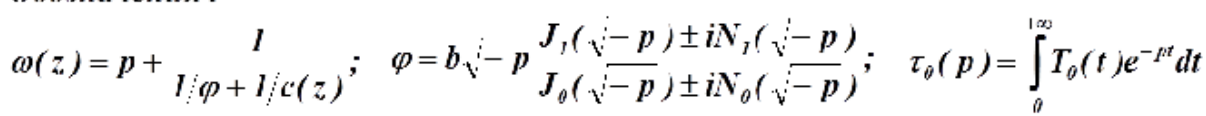

На рис. 2 представлены результаты прогноза изменения температуры воздуха по длине рудника с интервалом в 1 год после 10 лет эксплуатации по формулам (5) и (2). Сравнительный анализ графиков выявил значительные количественные расхождения результатов [7]. Согласно расчётам по методу Воропаева (2), воздух прогревает массив 
намного быстрее. Интенсивность прогревания отличается примерно в 5-8 раз. Так через 10 лет на расстоянии 10 км температура должна подняться на 1/4 градуса в (5) и почти на 2 градуса в (2). Также большую погрешность даёт использование коэффициента нестационарного теплообмена для расчёта быстрых теплообменных процессов, протекающих на интервалах времени порядка 1 часа.
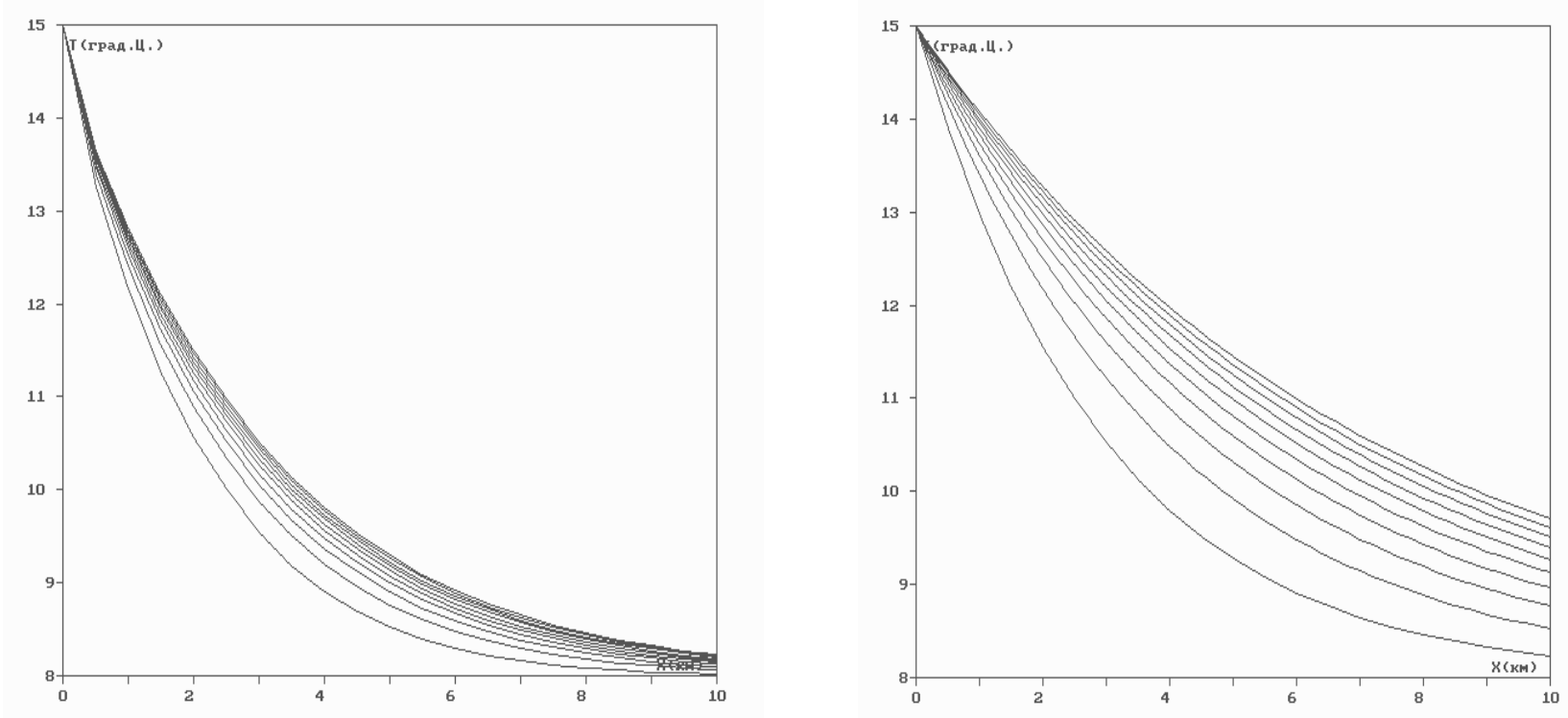

Рис. 2. Расчётные (слева по формуле (5), справа по формуле (2)) изменения температуры воздуха по длине выработки спустя 10 лет эксплуатации рудника с интервалом в 1 год

Представленный вычислительный метод относится к классу аналитических моделей, но позволяет получать результаты, не уступающие по точности имитационным в рамках постановочных условий. К недостаткам следует отнести его плохую адаптацию к добавлению осложняющих факторов. Например, введение в модель двухслойного массива с разными теплопроводностями слоёв (крепь ствола), увеличивает сложность итоговых зависимостей на порядок [8], что лишает их основного «аналитического» преимущества перед имитацией.

\section{Имитационное моделирование теплообмена методом конечных объёмов}

Возможностей аналитических методов становится недостаточно при необходимости комплексного учёта большого количества разнородных факторов, влияющих на распространение тепла в массиве и воздухе. К числу этих факторов относятся: отсутствие симметрии задачи, неоднородность и неизотропность термодинамических свойств пород, наличие влагообменных процессов и источников выделения тепла, нестационарное и не одномерное движение воздушного потока [9]. В подобной постановке задача теплообмена может быть решена только с помощью численных методов имитационного моделирования (рис. 3).

Система уравнений теплопереноса (6) решается численно методом конечных объёмов и позволяет получить решение с заданной точностью [10]. Несомненным преимуществом данного метода является вариативность постановочных условий. Отсутствие аналитических упрощений и преобразований позволяет без проблем добавлять в модель дополнительные факторы и производить расчёт тем же методом с учётом этих факторов. К недостаткам следует отнести большую вычислительную ресурсоёмкость, потерю математической наглядности и невозможность выявления аналитической функциональности исследуемого процесса. 


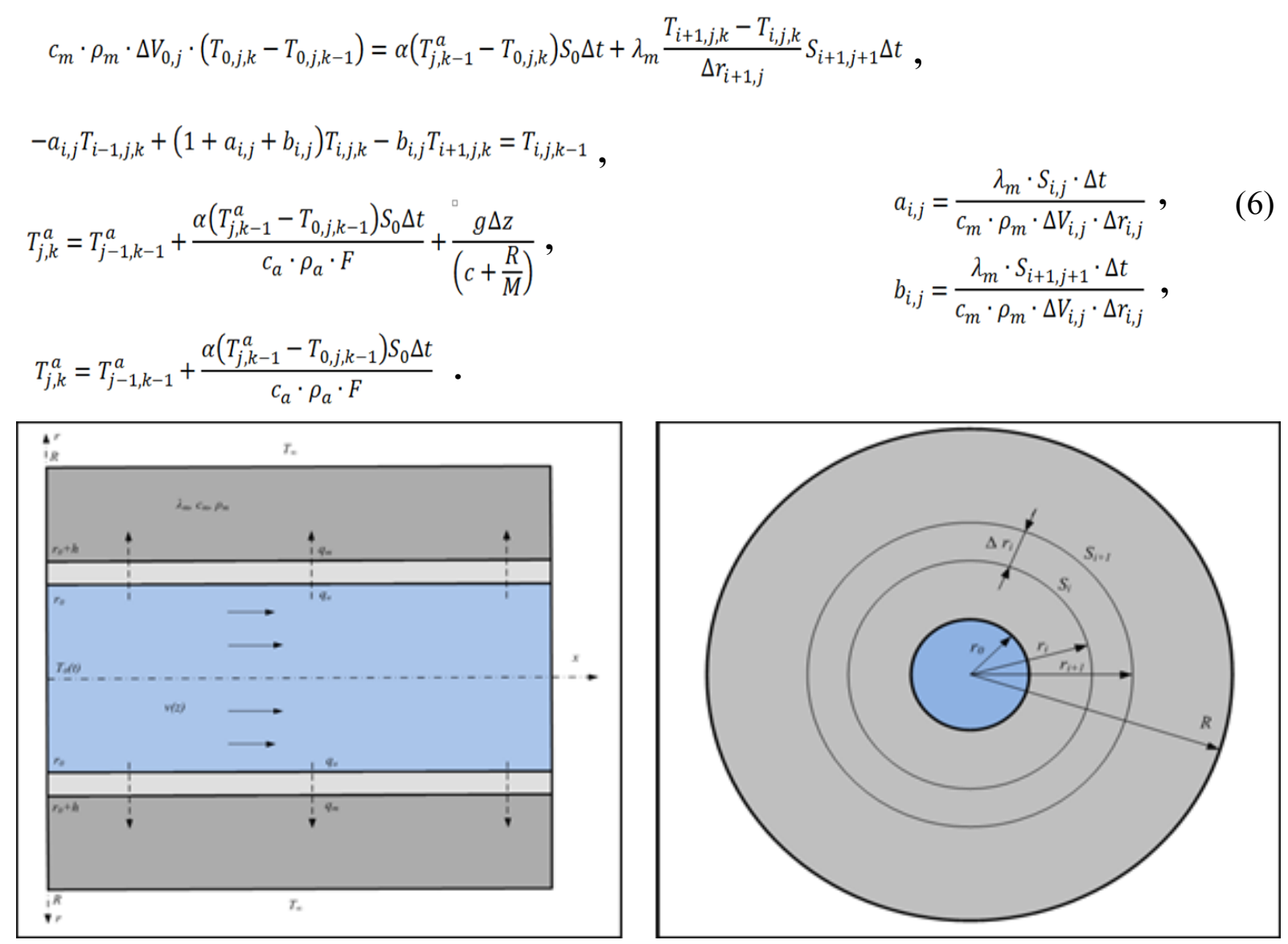

Рис. 3. Теплообмен между воздухом и породным массивом в горной выработке с учётом следующих факторов: влияние гидростатического нагрева/охлаждения воздуха в вертикальных и наклонных горных выработках; двухслойная структура окружающего выработку массива, представляющая систему «воздух - крепь - горные породы»; влияние тепловыделений фазовых переходов в рудничной атмосфере.

\section{Заключение}

На основании проведённого сравнительного анализа методов моделирования теплообменных процессов в шахтной теплофизике можно заключить, что аналитические и имитационные модели не противоречат, а дополняют друг друга, в целом давая наиболее полную, точную и понятную картину. Первые не требуют больших вычислительных ресурсов и дают простые аналитические зависимости, удобные для проведения инженерных расчётов. Вторые позволяют проводить детальное моделирование с учётом большого количества факторов и получать более точные и соответствующие реальности результаты. Поэтому оптимальным является комплексное использование методов аналитического и имитационного моделирования с целью извлечения преимуществ обоих методов [11].

Работа выполнена при поддержке гранта по конкурсу фундаментальных исследований Уральского отделения Российской академии наук (проект № 18-5-5-5).

\section{БИБЛИОГРАФИЧЕСКИЙ СПИСОК}

1. Щербань А.Н., Кремнёв О.А. Научные основы расчёта и регулирования теплового режима глубоких шахт: В 2-х т. - Киев: Изд-во АН УССР, 1959. - Т. 1. - С. 430.

2. Щербань А.Н. Основы теории и методы тепловых расчётов рудничного воздуха. - М.; Харковь: Углетехиздат, 1953. - С. 221. 
3. Воропаев А.Ф. Теория теплообмена рудничного воздуха и горных пород в глубоких шахтах. - М.: Недра, 1966. - С. 219.

4. Шалимов А.В., Кормщиков Д.С., Газизуллин Р.Р., Сёмин М.А. Моделирование динамики тепловых депрессий и ее влияния на проветривание горных выработок // Вестн. ПНИПУ: Геология. Нефтегазовое и горное дело. - 2014. - Т. 13, № 12. - C.41-47. DOI: 10.15593/2224-9923/2014.12.5

5. Казаков Б.П., Шалимов А.В., Сёмин М.А., Гришин Е.Л., Трушкова Н.А. Конвективная стратификация воздушных потоков по сечению горных выработок, ее роль в формировании пожарных тепловых депрессий и влияние на устойчивость проветривания // Горный журнал. - 2014. - №12. - С. 105-109.

6. Соболев С.Л. Уравнения математической физики. - 2-е изд., перераб.- М.; Л.: Гос. изд-во техн.теорет. лит., 1950. - С. 424.

7. Казаков Б.П., Левин Л.Ю., Шалимов А.В. Теория и практика прогнозирования, профилактики и борьбы с аварийными нарушениями проветривания рудников. - М.: ООО «Издательский дом Недра», 2016. - С. 244.

8. Казаков Б.П., Шалимов А.В., Гришин Е.Л. Теплообмен вентиляционного воздуха с крепью воздухоподающего ствола и породным массивом // Физико-технические проблемы разработки полезных ископаемых. - 2011. - № 5. - С. 92-98.

9. Левин Л.Ю., Семин М.А., Зайцев А.В. Численное решение сопряженной задачи теплораспределения в рудничной атмосфере и окружающем породном массиве для сети горных выработок произвольной топологии // Горный информационно-аналитический бюллетень (научно-технический журнал). 2013. - № 8. - С. 176-180.

10. Левин Л.Ю., Семин М.А., Зайцев А.В. Разработка математических методов прогнозирования микроклиматических условий в сети горных выработок произвольной топологии // Физико-технические проблемы разработки полезных ископаемых. - 2014. - № 2. - С. 154-161.

11. Бабина О.И. Сравнительный анализ имитационных и аналитических моделей // Имитационное моделирование. Теория и практика: материалы конф. сб. докл. - СПБ., 2009. - С. 73-77.

УДК 622.831

DOI:10.7242/echo.2020.2.21

\title{
МЕХАНИЗМ ОБРАЗОВАНИЯ ОЧАГОВ ГАЗОДИНАМИЧЕСКИХ ЯВЛЕНИЙ В СКЛАДЧАТЫХ СТРУКТУРАХ КАЛИЙНЫХ ПЛАСТОВ
}

\author{
Е.В. Лукьянец, Д.А. Бобров \\ Горный институт УрО РАН, г. Пермь
}

\begin{abstract}
Аннотация. Высокая степень изученности процесса газодинамических явлений не вызывает сомнений, но, к сожалению, в настоящее время для условий калийных рудников на Верхнекамском месторождении калийно-магниевых солей отсутствует понимание механизма образования очагов газодинамических явлений в складчатых структурах. Анализ геологических и горнотехнических условий проявления газодинамических явлений позволил выявить приуроченность очагов газодинамических явлений к зонам развития интенсивной складчатости и антиклинальным складкам в калийных пластах. Таким образом, представленная проблема образования очагов газодинамических явлений в складчатых структурах изучена недостаточно глубоко, и ее решение приобретает особую актуальность, имеет большое теоретическое и практическое значение. Разработан механизм образования очагов газодинамических явлений в зонах складчатых структур калийных пластов, заключающийся в формировании «газового» коллектора и области пониженной прочности соляных пород при образовании трещин складок и трещин отслоений с последующей фильтрацией свободного газа в образовавшиеся в складках пустоты из вмещающегося массива и сохранение образовавшихся газонасыщенных областей в соляном породном массиве как квазиизолированных систем пустот длительное геологическое время при условии превышения величиной начального градиента давления величины давления газа на контуре очага. Представленная совокупность трещин и расслоений, образовавшихся в пределах сформировавшейся складчатой структуры и заполненных свободным газом под давлением, представляет собой потенциальный очаг газодинамического явления, сформировавшийся в соляном породном массиве. Разработана модельная схема механизма образования очагов газодинамических явлений в складчатых структурах калийных пластов. Разработанный механизм образования очагов газодинамических явлений и его модельная схема приведут к повышению безопасности ведения горных работ в условиях складчатого строения породного массива на рудниках Верхнекамском месторождении калийно-магниевых солей за счет целенаправленного применения профилактических мероприятий.
\end{abstract}

